\title{
New exclusion limits for dark gauge forces from beam-dump data
}

\author{
Johannes Blümlein ${ }^{\mathrm{a}, *}$, Jürgen Brunner ${ }^{\mathrm{a}, \mathrm{b}}$ \\ a Deutsches Elektronen-Synchrotron, DESY, Platanenallee 6, D-15738 Zeuthen, Germany \\ b CPPM, Aix-Marseille Université, CNRS/IN2P3, Marseille, France
}

\section{A R T I C L E I N F O}

\section{Article history:}

Received 14 April 2011

Received in revised form 17 May 2011

Accepted 18 May 2011

Available online 26 May 2011

Editor: L. Rolandi

\section{Keywords:}

Dark gauge forces

Light shining through walls

Proton beam dump

Exclusion limits

\begin{abstract}
A B S T R A C T
We re-analyze published proton beam dump data taken at the U70 accelerator at IHEP Serpukhov with the $v$-calorimeter I experiment in 1989 to set mass-coupling limits for dark gauge forces. The corresponding data have been used for axion and light Higgs particle searches in Blümlein et al. (1991, 1992) [1,2] before. We determine new mass and coupling exclusion bounds for dark gauge bosons.
\end{abstract}

(c) 2011 Elsevier B.V. All rights reserved.

\section{Introduction}

Long range forces based on a $U(1)$ gauge symmetry beyond those of the $S U(3)_{C} \times S U(2)_{L} \times U(1)_{Y}$ Standard Model may exist yet unnoticed if their coupling to ordinary matter is very weak [3,4], see also [5]. Symmetries of this kind are discussed in various extensions of the Standard Model, see the surveys [6-8]. A new $U(1)$ gauge boson $\gamma^{\prime}$ with masses $m_{\gamma^{\prime}}$ in the $\mathrm{MeV}-\mathrm{GeV}$ range extends the Lagrangian of the Standard Model $\mathcal{L}_{\mathrm{SM}}$ to $[6,9,10]$

$$
\begin{aligned}
\mathcal{L}= & \mathcal{L}_{\mathrm{SM}}-\frac{1}{4} X_{\mu \nu} X^{\mu \nu}+\frac{\epsilon}{2} X_{\mu \nu} F^{\mu \nu}+e_{\psi} \epsilon \bar{\psi} \gamma_{\mu} \psi X^{\mu} \\
& +\frac{m_{\gamma^{\prime}}^{2}}{2} X_{\mu} X^{\mu}
\end{aligned}
$$

Here $X^{\mu}$ denotes the new vector potential and $X^{\mu \nu}=\partial^{\mu} X^{\nu}-$ $\partial^{v} X^{\mu}$ the corresponding field strength tensor, with $F^{\mu \nu}$ the $U(1)_{Y}$ field strength tensor. The mixing of the new $U(1)$ and $U(1)_{Y}$ of the Standard Model is induced by loops of heavy particles coupling to both fields $[3-5,8]$. The field $X_{\mu}$ is assumed to couple minimally to all charged Standard Model fermions $\psi$, with effective charge $e_{\psi} \epsilon$, where $e_{\psi}$ is the fermionic charge under $U(1)_{Y}$. For the generation of the mass term we assume the Stueckelberg formalism [11], as an example. Possible other mechanisms consist in technicolor or spontaneous symmetry breaking. The latter ones would lead to more terms in (1). The parameter $\epsilon$ denotes the mixing pa-

\footnotetext{
* Corresponding author.

E-mail address: Johannes.Bluemlein@desy.de (J. Blümlein).
}

rameter of the two $U(1)$ groups and may take values in the range $\epsilon \sim 10^{-23}-10^{-2}$, depending on the respective model, cf. [6].

Dark $U(1)$ gauge forces contribute to the anomalous magnetic moments of the electron and muon. Potential signals may be measured from $\Upsilon(3 S)$ decays. The $\gamma^{\prime}$ particles may be created in electron- and proton-beam dumps. So far signals of these particles have not been detected leading to various exclusion bounds in the $m_{\gamma^{\prime}}-\epsilon$ plane in the range of $\epsilon \in\left[5 \times 10^{-9}, 10^{-2}\right]$ and a series of mass regions in $m_{\gamma^{\prime}} \in\left[2 m_{e}, 3 \mathrm{GeV}\right]$, cf. e.g. Refs. [6-8,12].

In the present Letter we derive new exclusion bounds on dark $\gamma^{\prime}$ bosons using proton beam dump data at $p \sim 70 \mathrm{GeV}$. These data have been used in axion [13] and light Higgs boson searches, cf. $[1,2,14]$ in the past. On the experimental side, the present analysis thoroughly relies on the published data and results in Refs. [1,2]. We first describe the production process and the experimental facility, and then derive new mass and coupling bounds.

\section{The production process}

The abundant production of $\pi^{0}$ mesons in proton beam dumps leads to a production rate of $\gamma^{\prime}$

$$
\begin{aligned}
& \sigma\left(p p \rightarrow \gamma^{\prime} X\right) \\
& \quad=2 \epsilon^{2}\left(1-\frac{m_{\gamma^{\prime}}^{2}}{m_{\pi^{0}}^{2}}\right)^{3} \operatorname{Br}\left(\pi^{0} \rightarrow \gamma \gamma\right) \sigma\left(p p \rightarrow \pi^{0} X\right),
\end{aligned}
$$

through $\pi^{0} \rightarrow \hat{\gamma} \hat{\gamma}$, with the mixture $\hat{\gamma}=\left(\gamma+\epsilon \gamma^{\prime}\right) /(1+\epsilon)$ and $\operatorname{Br}\left(\pi^{0} \rightarrow \gamma \gamma\right)=0.98823 \pm 0.00034$ [15], neglecting contributions 
in higher powers of $\epsilon$. Here the phase space factor of the 2-particle decay has been accounted for [17]. The mass range of the produced $\gamma^{\prime}$ is limited by $m_{\gamma^{\prime}}<m_{\pi^{0}}=134.976 \mathrm{MeV}$ [15]. In principle this range may be expanded to higher meson decay thresholds including $\eta, \rho^{ \pm}, \omega$, and $\eta^{\prime}$ production, since the decay spectra of these particles contain a large fraction of photons, see also [10, 18]. However, one has to know the corresponding differential meson production spectra for $p A$ scattering in detail, which to our knowledge have not been measured in the energy region under consideration.

We consider the collision process $p F e \rightarrow \pi^{0} X$ at a momentum of the incoming proton of $p=68.6 \mathrm{GeV}$. The differential scattering cross sections for the reactions $p p \rightarrow \pi^{ \pm} X$ for values of $p_{\perp} \lesssim 1 \mathrm{GeV}$ were measured [19]. One may use the representation

$$
\begin{aligned}
& E \frac{d^{2} \sigma\left(p p \rightarrow \pi^{0} X\right)}{d x_{F} d p_{\perp}^{2}} \\
& \quad=\frac{1}{2}\left[E \frac{d^{2} \sigma\left(p p \rightarrow \pi^{+} X\right)}{d x_{F} d p_{\perp}^{2}}+E \frac{d^{2} \sigma\left(p p \rightarrow \pi^{-} X\right)}{d x_{F} d p_{\perp}^{2}}\right]
\end{aligned}
$$

for the invariant cross sections, which is even a slight underestimation w.r.t. multiplicity measurements, cf. Section 6, Ref. [21]. The differential cross sections were parameterized by

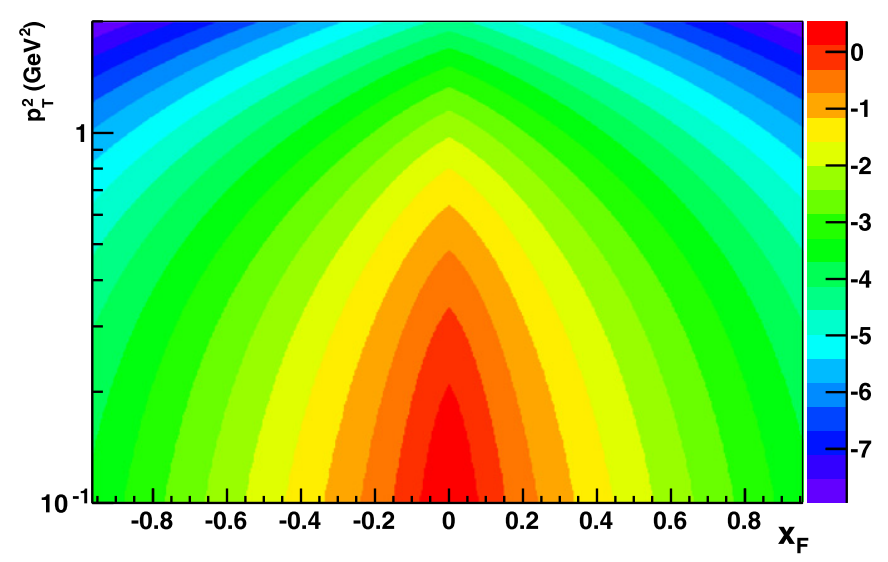

Fig. 1. $p_{\perp}^{2}$ vs. $x_{F}$ distribution of the $\pi^{0}$ production spectrum in $\mathrm{p}-\mathrm{Fe}$ collisions at $p=68.6 \mathrm{GeV}$. The color scale is in $\log _{10}$. (For interpretation of the references to color in this figure, the reader is referred to the web version of this Letter.)

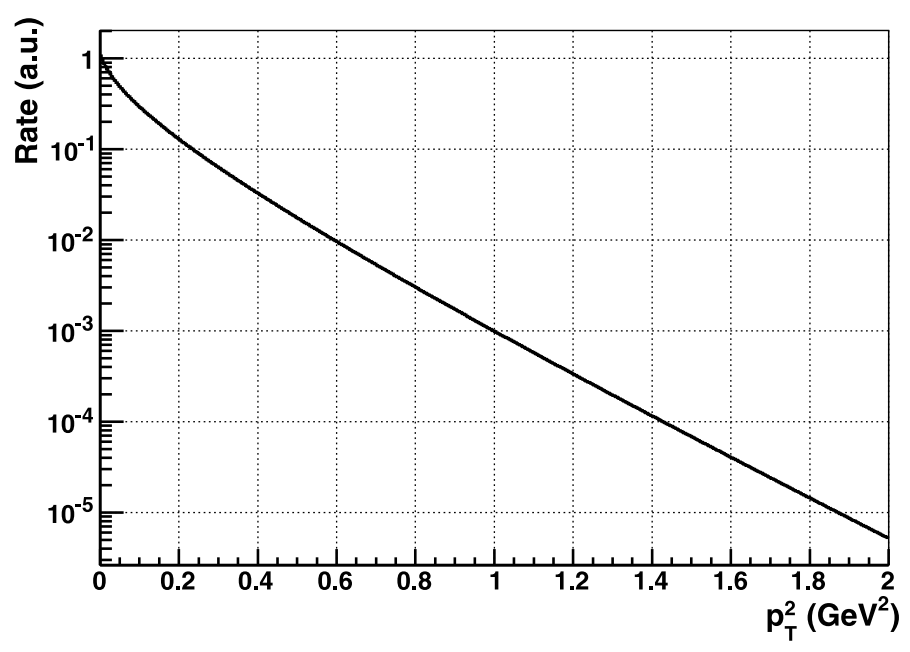

$$
\begin{aligned}
& E \frac{d^{2} \sigma\left(p p \rightarrow \pi^{ \pm} X\right)}{d x_{F} d p_{\perp}^{2}} \\
& \quad=A_{ \pm} \exp \left(B_{ \pm}\left|x_{F}\right|+C_{ \pm} x_{F}^{2}\right) \exp \left(D_{ \pm} p_{\perp}+E_{ \pm} p_{\perp}^{2}\right)
\end{aligned}
$$

in [19]. Here $x_{F}=p_{L} / p_{L}^{\max }$ denotes Feynman- $x$ and $p_{\perp}$ the transverse momentum. The normalizations obey $A_{+} / A_{-}=2.16 \pm 0.24$ and the other parameters are presented in Table 1.

Here we refer for the $p_{\perp}$ distribution to the values of $\left\langle p_{\perp}\right\rangle=$ $0.2 \mathrm{GeV}$. The resulting distributions in $p_{\perp}^{2}$ and $x_{F}$ are shown in Figs. 1, 2.

The actual beam dump experiment used an iron target. The inclusive iron-proton cross section is related to the proton-proton cross section from (2) by

$\sigma\left(p F e \rightarrow \pi^{0} X\right)=A^{\alpha\left(x_{F}\right)} \sigma\left(p p \rightarrow \pi^{0} X\right)$,

with $A=56$. The $A^{\alpha\left(x_{F}\right)}$ dependence of the $\pi^{0}$-production cross section on nuclei as of iron can be parameterized by

$\left\langle\alpha\left(x_{F}\right)\right\rangle \simeq 0.55$,

based on the compilation given in [20] referring to Eq. (3). The inclusive cross section at $69 \mathrm{GeV}$ was measured with [21]

$\sigma\left(p p \rightarrow \pi^{0} X\right)=74 \pm 1.0 \mathrm{mb}$.

We generate the $\gamma^{\prime}$ particle production in the $p p$ center-ofmomentum system and boost to the laboratory system then. We investigate the mass range of $\gamma^{\prime}$ particles above the 2-electron threshold of $1.022 \mathrm{MeV}$ up to $m_{\pi^{0}}$. A cut $\left(p_{\perp} / p_{L}\right)_{\text {lab }}<\tan \left(\Theta_{\max }\right)$ is applied to ensure that the produced particle reaches the fiducial volume of the detector (see below). The effect of this cut is illustrated in Fig. 3 which shows the spectra of particles which pass the fiducial volume cut with respect to all produced particles. Results for $\pi^{0}$ are given as well as for $\gamma^{\prime}$ of various masses. The kinematic factor $\left(1-m_{\gamma^{\prime}}^{2} / m_{\pi^{0}}^{2}\right)^{3}$ from (2) is included for the $\gamma^{\prime}$. The only relevant decay channel in the considered mass range is $\gamma^{\prime} \rightarrow e^{+} e^{-}$. The inverse live time of the $\gamma^{\prime}$ particle is then given by [10]

Table 1

\begin{tabular}{lll}
\hline & + & - \\
\hline$B$ & $-5.21 \pm 1.03$ & $-9.51 \pm 0.21$ \\
$C$ & $-1.80 \pm 2.62$ & $+2.14 \pm 1.74$ \\
$D$ & $-1.80 \pm 0.31$ & $-1.22 \pm 0.38$ \\
$E$ & $-4.26 \pm 0.48$ & $-4.44 \pm 0.58$ \\
\hline
\end{tabular}

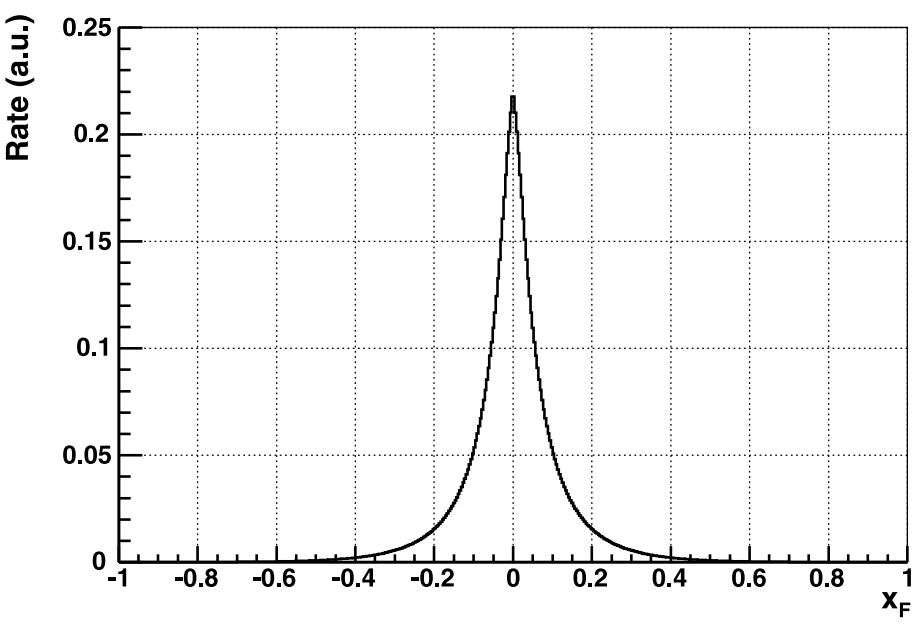

Fig. 2. The $p_{\perp}^{2}$ and $x_{F}$ distribution of the $\pi^{0}$ production spectrum in $\mathrm{p}-\mathrm{Fe}$ collisions at $p=68.6 \mathrm{GeV}$. 


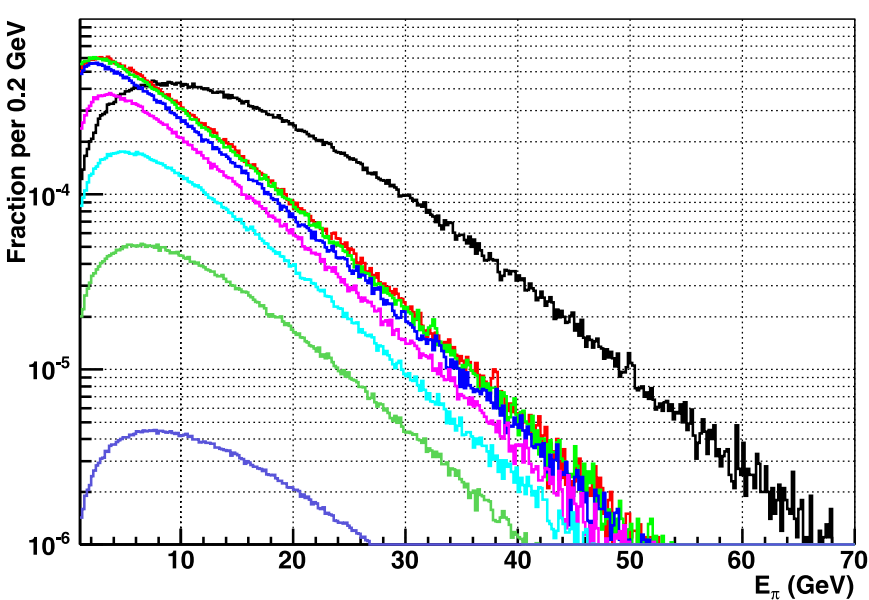

Fig. 3. Fraction of produced $\gamma^{\prime}$ particles which pass the fiducial volume cut as function of their energy in the laboratory frame. Colors: $\gamma^{\prime}$ with masses between 0 and $120 \mathrm{MeV}$ in steps of $20 \mathrm{MeV}$ (from top to bottom). The black line shows the corresponding $\pi^{0}$ for comparison. (For interpretation of the references to color in this figure, the reader is referred to the web version of this Letter.)

$\tau\left(\gamma^{\prime}\right)^{-1}=\frac{1}{3} \alpha_{Q E D} m_{\gamma^{\prime}} \epsilon^{2} \sqrt{1-\frac{4 m_{e}^{2}}{m_{\gamma^{\prime}}^{2}}}\left(1+\frac{2 m_{e}^{2}}{m_{\gamma^{\prime}}^{2}}\right)$.

Potential electron-pairs from $\gamma^{\prime}$ decay manifest as electromagnetic showers in the detector used. The mass and coupling limits on the $\gamma^{\prime}$ bosons given below are derived from the observed rate of these showers over the background, cf. [1].

\section{The experimental setup and data taking}

The beam dump experiment was carried out at the U70 accelerator at IHEP Serpukhov during a three months exposure in 1989. Data have been taken with the $v$-CAL I experiment, a neutrino detector. All technical details of this experiment have been described in [1] and a detailed description of the detector was given in [22]. Here we only summarize the key numbers which are crucial for the present analysis.

The target part of the detector is used as a fiducial volume to detect the decays of the $\gamma^{\prime}$. It has a modular structure and consists of 36 identical modules along the beam direction. Each of the modules is composed of a $5 \mathrm{~cm}$ thick aluminum plate, a pair of drift chambers to allow for three dimensional tracking, and a $20 \mathrm{~cm}$ thick liquid scintillator plane to measure the energy deposit of charged particles.

For the beam dump experiment a fiducial volume of 30 modules with a total length of $l=23 \mathrm{~m}$ is chosen, starting with the fourth module at a distance of $l_{\text {dump }}=64 \mathrm{~m}$ down-stream of the beam dump. The lateral extension of the fiducial volume is $2.6 \times 2.6 \mathrm{~m}^{2}$. In the following we use conservatively a slightly smaller fiducial volume, defined as a cone pointing to the beam dump with a ground circle of $2.6 \mathrm{~m}$ in diameter at the end of the fiducial volume, i.e. at a distance of $87 \mathrm{~m}$ from the dump. This leads to the following simple fiducial volume cut:

$\left(p_{\perp} / p_{L}\right)_{\text {lab }}<1.3 / 87=0.015$.

For particles which traverse the fiducial volume, the decay probability $w_{\text {dec }}$ for decays $\gamma^{\prime} \rightarrow e^{+} e^{-}$is then given by

$w_{\text {dec }}=\exp \left[-\frac{l_{\text {dump }}}{c \tau\left(\gamma^{\prime}\right)} \frac{m_{\gamma^{\prime}}}{p}\right]\left[1-\exp \left(\frac{l}{c \tau\left(\gamma^{\prime}\right)} \frac{m_{\gamma^{\prime}}}{p}\right)\right]$,

with $c$ the velocity of light, $m_{\gamma^{\prime}}$ and $p$ are the mass and momentum of the $\gamma^{\prime}$.

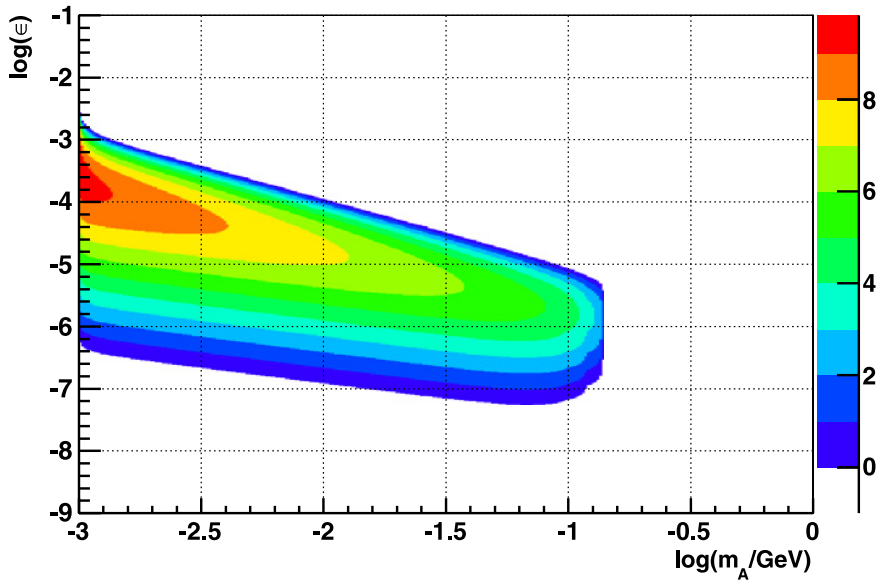

Fig. 4. Detected $\gamma^{\prime}$ events. Color scale in $\log _{10}$ from $10^{9}$ events (red) to one event (dark blue). (For interpretation of the references to color in this figure, the reader is referred to the web version of this Letter.)

During the three months exposure time in $19891.71 \times 10^{18}$ protons on target had been accumulated [1]. The signature of event candidates from $\gamma^{\prime} \rightarrow e^{+} e^{-}$is a single electromagnetic shower in beam direction. This signature is identical to the one from the axion or light Higgs particle decay search which was performed in [1]. For energy deposits above $3 \mathrm{GeV}$ in the detector the reconstruction code can distinguish electromagnetic from hadronic showers very well. Therefore the following final cuts for the selection of isolated electromagnetic showers had been chosen [1]:

- A minimal electromagnetic shower energy of $E_{\mathrm{elm}}>3 \mathrm{GeV}$;

- A maximal hadronic shower energy of $E_{\text {had }}<1.5 \mathrm{GeV}$;

- A maximal angle with respect to the beam direction of $\Theta_{\mathrm{elm}}<$ $0.05 \mathrm{rad}$.

From the total data sample of 3880 reconstructed events, 5 pass these cuts. Background estimates from the simulation of $v_{\mu}$ and $v_{e}$ interactions in the detector account for 3.5 events. The Poisson probability to observe 5 or less events for an expectation of 3.5 events is $86 \%$. Data are therefore compatible with the simulated background from conventional neutrino interactions.

\section{Search for decays $\gamma^{\prime} \rightarrow e^{+} e^{-}$}

Signals from $\gamma^{\prime} \rightarrow e^{+} e^{-}$decays pass the cuts mentioned above with an energy independent efficiency of $E=70 \%$ if the true energy of the decaying particle is above $3 \mathrm{GeV}$. The total number of expected signal events can therefore be calculated as

$N_{\text {sig }}=N_{\text {tot }} \times \frac{\sigma\left(p F e \rightarrow \gamma^{\prime} X\right)_{\text {forward }}}{\sigma(p p \rightarrow X)} \times w_{\text {dec }} \times E$,

with $N_{\text {tot }}$ the total number of protons on target during the exposure time. The index 'forward' indicates the application of the fiducial volume cut. The dependence of $N_{\text {sig }}$ on $m_{\gamma^{\prime}}$ and $\epsilon$ is shown in Fig. 4.

For $m_{\gamma^{\prime}} \approx 2 \mathrm{MeV}$ and $\epsilon \approx 2 \cdot 10^{-4}$ the decay probability reaches its maximum of $11 \%$ and more than $10^{9}$ signal events would be expected in the detector. For larger $\epsilon$ the decay length decreases exponentially and at some point all $\gamma^{\prime}$ particles decay before reaching the detector. For smaller values of $\epsilon$ the decay length increases. At $\epsilon<10^{-6}$ most of the particles pass the detector without decaying. For even smaller values of $\epsilon$ the event rate of both production and decay probability decrease proportional to $\epsilon^{2}$. The dependence of the iso-event number lines on $m_{\gamma^{\prime}}$ is governed by 


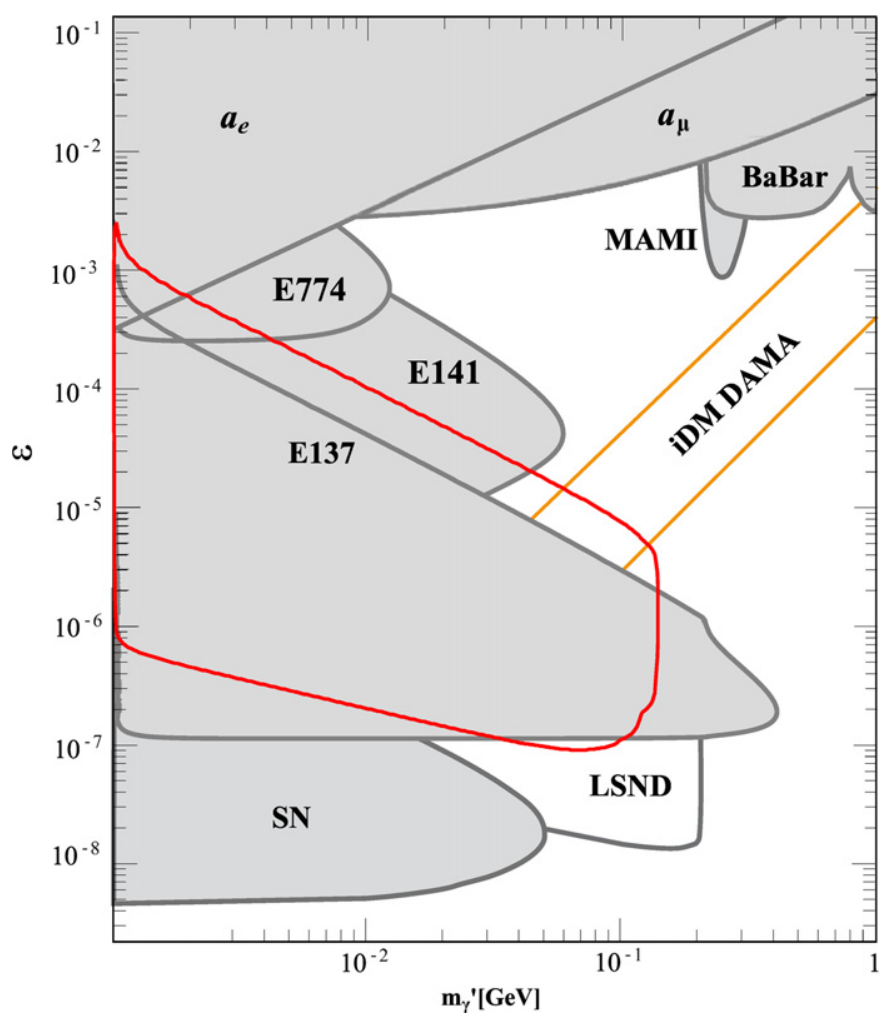

Fig. 5. Comparison of the present exclusion bounds (red line) with other limits from the measurement of the anomalous magnetic moments $a_{e}$ and $a_{\mu}$ [23], $\Upsilon(3 S)$ decay [24], results from MAMI [25], the beam dump experiments E137, E141, E774 [26-28], and supernovae cooling [6,29]. We indicate the prospects for LSND $[10,30]$ (open grey-bounded area), and the DAMA/LIBRA region (open orange bounded area) [31]. The limits for $\epsilon>10^{-7}$ have been taken from Ref. [8]. (For interpretation of the references to color in this figure, the reader is referred to the web version of this Letter.)

the boost factor $p / m_{\gamma^{\prime}}$. The sensitivity is kinematics limited to values $m_{\gamma^{\prime}}<m_{\pi^{0}}$.

Confindence limits are calculated with the CLs method [16] according to

$c=1-\sum_{n=0}^{N} P(n, s+b) / \sum_{n=0}^{N} P(n, b)$

with $P(n, x)$ the Poisson probability to observe $\mathrm{n}$ events for a mean value of $x$. For $N=5$ observed events and a background of $b=3.5$ we could exclude a signal contribution of 7.3 events at 95\% C.L. $(c=0.95)$. If we conservatively assume an uncertainty of a factor two for the background estimate $b$, the 95\% C.L. limit moves to 8.8 events. The corresponding exclusion region is shown as the red line in comparison with limits from other experiments in Fig. 5, see also $[6,8,10]$.

At large values of $\epsilon$ studies of the anomalous magnetic moments of the muon and electron [23], of rare decays of heavy mesons [24], and results from MAMI [25], put stringent limits. For $10^{-3}<\epsilon<10^{-7}$ beam dump experiments [26-28] give the best sensitivity. For even smaller values of $\epsilon$ limits can be derived by studying the dynamics of supernovae cooling [29]. For completeness, the prospects for the sensitivity of a reanalysis of LSND data [30] is also shown, as proposed in [10].

The present analysis is sensitive to the region $10^{-3}<\epsilon<10^{-7}$ and the exclusion region largely overlaps with the one from E137. However, we are sensitive to larger values of $\epsilon$ for the same values of $m_{\gamma^{\prime}}$. In particular, a new region around $\epsilon \approx 10^{-5}$ and $m_{\gamma^{\prime}} \approx$ $50 \mathrm{MeV}$ is explored here. This region is part of a band (shown in orange) which correlates $\epsilon$ and $m_{\gamma^{\prime}}$ within certain supersymmetric theories [32]. Within these models heavy dark matter candidate particles pick up an $U(1)^{\prime}$ charge and can scatter elastically through $\gamma^{\prime}$ exchange. This would allow to explain the annual modulation of the DAMA/LIBRA experiment [31]. For a more extensive discussion, see [6].

\section{Conclusions}

We have re-analyzed proton beam dump data taken at the U70 accelerator at IHEP Serpukhov with the $v$-calorimeter I experiment in 1989 to set mass and coupling limits for dark gauge forces, searching for electromagnetic signatures according to the decay $\gamma^{\prime} \rightarrow e^{+} e^{-}$. The analysis extends the region excluded by former experiments in the mass region $m_{\gamma^{\prime}} \in\left[0.03 \mathrm{GeV}, m_{\pi_{0}}\right]$ towards larger values in the mixing parameter $\epsilon \in\left[2 \times 10^{-6}, 2 \times 10^{-5}\right]$. A lower part of the anticipated DAMA/LIBRA region is excluded. At lower values of $\epsilon \approx 10^{-7}$ a smaller region of masses in the range $m_{\gamma^{\prime}} \in[0.03,0.1] \mathrm{GeV}$ is excluded beyond the bounds given by E137 [26]. In future experiments signals from dark gauge forces will be searched for in the yet unexplored regions shown in Fig. 5, see e.g. Ref. [8] for proposals.

\section{Acknowledgements}

We would like to thank our former colleagues of the $v$-Cal I experiment for collaboration during the time 1983-1990. For discussions we thank M. Walter and A. Ringwald. This work has been supported in part by DFG Sonderforschungsbereich Transregio 9, Computergestützte Theoretische Teilchenphysik and EU Network LHCPHENOnet PITN-GA-2010-264564.

\section{References}

[1] J. Blümlein, J. Brunner, H.J. Grabosch, P. Lanius, S. Nowak, C. Rethfeldt, H.E. Ryseck, M. Walter, et al., Z. Phys. C 51 (1991) 341.

[2] J. Blümlein, J. Brunner, H.J. Grabosch, P. Lanius, S. Nowak, C. Rethfeldt, H.E. Ryseck, M. Walter, et al., Int. J. Mod. Phys. A 7 (1992) 3835.

[3] P. Fayet, Phys. Lett. B 96 (1980) 83; P. Fayet, Nucl. Phys. B 187 (1981) 184.

[4] B. Holdom, Phys. Lett. B 166 (1986) 196.

[5] P. Fayet, Nucl. Phys. B 347 (1990) 743; P. Fayet, Phys. Lett. B 675 (2009) 267, arXiv:0812.3980 [hep-ph].

[6] J.D. Bjorken, R. Essig, P. Schuster, N. Toro, Phys. Rev. D 80 (2009) 075018 arXiv:0906.0580 [hep-ph].

[7] J. Redondo, A. Ringwald, arXiv:1011.3741 [hep-ph].

[8] S. Andreas, A. Ringwald, arXiv:1008.4519 [hep-ph].

[9] D. Feldman, B. Kors, P. Nath, Phys. Rev. D 75 (2007) 023503, arXiv:hep-ph/ 0610133;

D. Feldman, Z. Liu, P. Nath, Phys. Rev. D 75 (2007) 115001, arXiv:hep-ph/ 0702123.

[10] R. Essig, R. Harnik, J. Kaplan, N. Toro, Phys. Rev. D 82 (2010) 113008 arXiv:1008.0636 [hep-ph].

[11] E.C.G. Stueckelberg, Helv. Phys. Acta 11 (1938) 225.

[12] S. Davidson, S. Hannestad, G. Raffelt, JHEP 0005 (2000) 003, arXiv:hep-ph/ 0001179;

M. Glück, S. Rakshit, E. Reya, Phys. Rev. D 76 (2007) 091701, arXiv:hep-ph/ 0703140;

S.L. Adler, J. Gamboa, F. Mendez, J. Lopez-Sarrion, Annals Phys. 323 (2008) 2851, arXiv:0801.4739 [hep-ph].

[13] P. Fayet, Introduction to axions, in: Proceedings of the Conference "QCD and Lepton Physics", vol. 1, Ecole Norm. Sup., Paris, 1981, pp. 307-313, LPTENS 8108;

R.D. Peccei, Adv. Ser. Direct. High Energy Phys. 3 (1989) 503;

R.D. Peccei, Lect. Notes Phys. 741 (2008) 3, arXiv:hep-ph/0607268;

J. Blümlein, On the experimental status of light pseudo-scalar particles, in: Proceedings of the High Energy Physics Workshop, Georgenthal, Leipzig University Press, Germany, 1984, p. 100;

P. Sikivie, Nucl. Phys. B (Proc. Suppl.) 87 (2000) 41, arXiv:hep-ph/0002154; J.E. Kim, G. Carosi, Rev. Mod. Phys. 82 (2010) 557, arXiv:0807.3125 [hep-ph] 
[14] J. Blümlein, R. Nahnhauer, S. Nowak, S. Schlenstedt, M. Walter, Estimation of the axion determination rate via the decay $a^{0} \rightarrow \gamma \gamma$ in a proton beam-dump experiment at the U-70-Accelerator, Internal note, IfH AdW, January 1984, 31 pp. (unpublished).

[15] K. Nakamura, et al., Particle Data Group, J. Phys. G 37 (2010) 075021 http://pdg.lbl.gov/.

[16] G. Zech, Nucl. Instr. Meth. A 277 (1989) 608.

[17] B. Batell, M. Pospelov, A. Ritz, Phys. Rev. D 80 (2009) 095024, arXiv:0906.5614 [hep-ph].

[18] L. Barabash, S. Baranov, Y. Batusov, S. Bunyatov, O. Denisov, A. Karev, M. Kazarinov, O. Klimov, et al., Phys. Lett. B 295 (1992) 154; L. Barabash, S. Baranov, Y. Batusov, S. Bunyatov, O. Denisov, A. Karev, M. Kazarinov, O. Klimov, et al., Sov. J. Nucl. Phys. 55 (1992) 1810.

[19] V.V. Ammosov, et al., France-Soviet Union Collaboration, Nuovo Cim. A 40 (1977) 237.

[20] D.S. Barton, G.W. Brandenburg, W. Busza, T. Dobrowolski, J.I. Friedman, C. Halliwell, H.W. Kendall, T. Lyons, et al., Phys. Rev. D 27 (1983) 2580.

[21] M. Boratav, et al., France-Soviet Union Collaboration, Nucl. Phys. B 111 (1976) 529;

H. Blumenfeld, et al., France-Soviet Union Collaboration, Phys. Lett. B 45 (1973) 525

[22] L.S. Barabash, S.A. Baranov, Y.A. Batusov, S.A. Bunyatov, V.Y. Valuev, I.A. Golutvin, O.Y. Denisov, M.Y. Kazarinov, et al., Instrum. Exp. Tech. 46 (2003) 300.

[23] M. Pospelov, Phys. Rev. D 80 (2009) 095002, arXiv:0811.1030 [hep-ph].
[24] R. Essig, P. Schuster, N. Toro, Phys. Rev. D 80 (2009) 015003, arXiv:0903.3941 [hep-ph];

B. Aubert, et al., BaBar Collaboration, arXiv:0902.2176 [hep-ex].

[25] H. Merkel, et al., A1 Collaboration, arXiv:1101.4091 [nucl-ex].

[26] J.D. Bjorken, S. Ecklund, W.R. Nelson, A. Abashian, C. Church, B. Lu, L.W. Mo, T.A. Nunamaker, et al., E137 Collaboration, Phys. Rev. D 38 (1988) 3375.

[27] E.M. Riordan, M.W. Krasny, K. Lang, P. De Barbaro, A. Bodek, S. Dasu, N. Varelas, X. Wang, et al., E141 Collaboration, Phys. Rev. Lett. 59 (1987) 755.

[28] A. Bross, M. Crisler, S.H. Pordes, J. Volk, S. Errede, J. Wrbanek, E774 Collaboration, Phys. Rev. Lett. 67 (1991) 2942.

[29] M.S. Turner, Phys. Rev. Lett. 60 (1988) 1797.

[30] C. Athanassopoulos, et al., LSND Collaboration, Phys. Rev. C 58 (1998) 2489, arXiv:nucl-ex/9706006; L.B. Auerbach, et al., LSND Collaboration, Phys. Rev. Lett. 92 (2004) 091801, arXiv:hep-ex/0310060.

[31] R. Bernabei, P. Belli, F. Cappella, R. Cerulli, C.J. Dai, A. d'Angelo, H.L. He, A. Incicchitti, et al., Eur. Phys. J. C 67 (2010) 39, arXiv:1002.1028 [astro-ph.GA].

[32] K.R. Dienes, C.F. Kolda, J. March-Russell, Nucl. Phys. B 492 (1997) 104, arXiv:hep-ph/9610479;

C. Cheung, J.T. Ruderman, L.-T. Wang, I. Yavin, Phys. Rev. D 80 (2009) 035008 , arXiv:0902.3246 [hep-ph];

D.E. Morrissey, D. Poland, K.M. Zurek, JHEP 0907 (2009) 050, arXiv:0904.2567 [hep-ph]. 